\title{
Neudesin as a unique secreted protein with multi-functional roles in neural functions, energy metabolism, and tumorigenesis
}

\author{
Hiroya Ohta ${ }^{1}$, Ikuo Kimura ${ }^{2}$, Morichika Konishi ${ }^{1}$ and Nobuyuki Itoh ${ }^{3 *}$ \\ ${ }^{1}$ Department of Microbial Chemistry, Kobe Pharmaceutical University, Kobe, Japan, ${ }^{2}$ Department of Applied Biological \\ Science, Graduate School of Agriculture, Tokyo University of Agriculture and Technology, Tokyo, Japan, ${ }^{3}$ Department of \\ Genetic Biochemistry, Kyoto University Graduate School of Pharmaceutical Sciences, Kyoto, Japan
}

Neudesin was originally identified as a secreted protein with neurotrophic activity, and, thereafter, was also termed neuron-derived neurotrophic factor (NENF) or the candidate oncogene GIG47. Neudesin with a conserved cytochrome 5-like heme/steroid-binding domain activates intracellular signaling pathways possibly through the activation of $G$ protein-coupled receptors. In the brain, hypothalamic Neudesin decreases food intake. Neudesin knockout (KO) mice also exhibit anxiety-like behavior, indicating its roles in the hippocampal anxiety circuitry. Neudesin is also expressed in various peripheral tissues. Neudesin KO mice are strongly resistant to high-fat diet (HFD)-induced obesity due to elevated systemic sympathetic activity, heat production, and adipocytic lipolysis. Neudesin, which is over-expressed or induced by DNA hypomethylation in multiple human cancers, also stimulates tumorigenesis. These findings indicate that Neudesin plays roles in neural functions, energy metabolism, and tumorigenesis and is expected to be a novel target for obesity and anti-cancer treatments.

Specialty section:

This article was submitted to

Cellular Biochemistry,

a section of the journal

Frontiers in Molecular Biosciences

Received: 29 March 2015 Accepted: 04 May 2015

Published: 19 May 2015

Citation:

Ohta H, Kimura I, Konishi M and Itoh $N$ (2015) Neudesin as a unique secreted protein with multi-functional roles in neural functions, energy metabolism, and tumorigenesis.

Front. Mol. Biosci. 2:24. doi: 10.3389/fmolb.2015.00024
Keywords: anxiety, membrane-associated progesterone receptor, metabolism, neudesin, neuron-derived

\section{Introduction}

Neudesin was originally identified as a secreted protein with neurotrophic activity from mouse embryos (Kimura et al., 2005). Thereafter, it was also termed NENF and GIG47 (Han et al., 2012; Byerly et al., 2013). Human Neudesin is a secreted protein of 172 amino acids with a conserved cytochrome 5-like heme/steroid-binding domain of $\sim 100$ amino acids (Figure 1A) (Kimura et al., 2005, 2008). Neudesin is a member of the membrane-associated progesterone receptor (MAPR) protein family comprising three additional members with characteristic cytochrome 5-like heme/steroid-binding domains: progesterone receptor-membrane component (PGRMC1), PGRMC2, and Neuferricin (Figure 1B) (Ohta and Itoh, 2012; Kimura et al., 2013).

PGRMC1 was originally identified as a putative receptor for progesterone (Meyer et al., 1996). However, PGRMC1 binds heme, not progesterone (Cahill, 2007). PGRMC1 and PGRMC2 are mainly located in the endoplasmic reticulum (Gerdes et al., 1998; Chen et al., 2010). The expression of PGRMC1 is up-regulated in cancer cells. PGRMC1 promotes cell survival and damage resistance in cancer cells and also plays roles in lipid, drug, and hormone metabolism in the liver and neuroprotection in the brain. PGRMC2 is also expressed in breast adenocarcinoma neurotrophic factor, obesity, tumorigenesis 
A

Neudesin
1 MVGPAPRRRLRPLAALALVLALAPGLPTARAGQTPRPAERGPPVRLFTEEELARYGGEEE $*$
DQPIYLAVKGVVFDVTSGKEFYGRGAPYNALTGKDSTRGVAKMSLDPADLTHDTTGLTAK 172 ELEALDEVFTKVYKAKYPIVGYTARRILNEDGSPNLDFKPEDQPHFDIKDEF
B

PGRMC1

PGRMC2

Neudesin

Neuferricin
FTPAELRRFDG-VQDPRILMAINGKVFDVTKGRKFYGPEG--PYGVFAGRDASRGLATFCLDKE

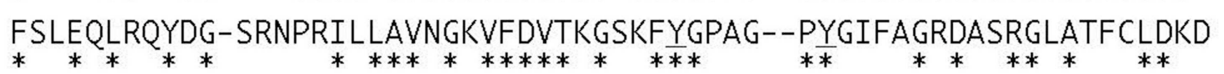
FTEEELARYGGEEEDQPIYLAVKGVVFDVTSGKEFYGRGA--PYNALTGKDSTRGVAKMSLDPA

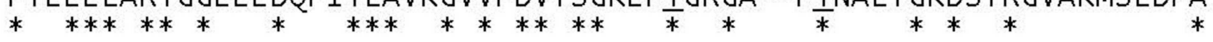
FIPEELSRYRGGPGDPGLYLALLGRVYDVSSGRRHYEPGS--HYSGFAGRDASRAFVTGDCSEA

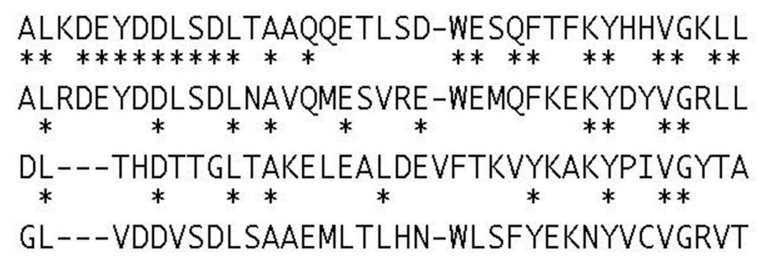

FIGURE 1 | Amino acid sequence of human Neudesin (A) and comparison of heme/steroid-binding domains in the human MAPR family (B). (A) Underlines indicate a cleavable secreted signal sequence and conserved heme/steroid-binding domain. Asterisks indicate the two conserved tyrosine residues, 82 and 88 , which are essential for heme-binding. The numbers refer to amino acid positions. (B) Dashes indicate gaps introduced to align sequences. Asterisks indicate identical amino acid residues in sequences. Underlines indicate the two conserved tyrosine residues essential for heme-binding in the MAPR family.
(Rohe et al., 2009; Intlekofer and Petersen, 2011). In contrast, Neuferricin is a secreted protein that promotes neurogenesis in neural precursor cells and suppresses cell survival in Neuro2a cells (Kimura et al., 2010). Neuferricin also enhances the survival of ectoside-exposed cells, cytochrome P450 activities, and the growth and survival of HeLa cells (Xie et al., 2011; Bruce and Rybak, 2014).

PGRMC1 and PGRMC2 have two conserved introns, indicating that they were generated from a common ancestral gene. In contrast, Neudesin has non-conserved introns. Thus, Neudesin is not evolutionarily related to the other members of the MAPR family (Ohta and Itoh, 2012). The roles and action mechanism of Neudesin are also distinct from those of the other members. Neudesin plays roles as a multi-functional secreted protein in neural functions, energy metabolism, and tumorigenesis. In this review, we focused on multi-functional Neudesin with recent findings.

\section{Structure}

The amino acid sequence of Neudesin is highly conserved in vertebrates (Ohta and Itoh, 2012). A nuclear magnetic resonance analysis indicated that Neudesin, which is also termed GIG47, has an $\alpha$-helice/ $\beta$-strand structure with a $\beta 1-\alpha 1-\beta 2-\beta 3-\alpha 2-\beta 4-\alpha 3-\alpha 4$ $\beta 5-\beta 6$ topology. The heme/steroid-binding domain is located in the $\alpha 2-\beta 4-\alpha 3$ topology. A homology modeling calculation with the known tertiary structure of 1TOG, a hypothetical protein of unknown function with a cytochrome b5-like fold, indicated the tertiary structure of Neudesin. A potential heme/steroidbinding hydrophobic pocket is visible between the $\alpha 2$ and $\alpha 3$ helices. Tyrosine residues 82 and 88 in this pocket are essential for heme-binding (Han et al., 2012).

\section{Activity and Action Mechanism of Neudesin}

Neudesin is preferentially expressed in the brain and spinal cord at embryonic stages in mice, but is widely expressed in various tissues including the brain, adipose tissue, heart, lung, and kidney at postnatal stages. In the brain, Neudesin is preferentially expressed in neurons. Neudesin exhibits significant neurotrophic activity in primary cultured neurons, but not mitogenic activity in primary cultured astrocytes, indicating that it is a neurotrophic factor. The conserved cytochrome 5-like heme/steroid-binding domain of Neudesin has been shown to bind heme and is required for its activity (Kimura et al., 2005, 2008). Neudesin activates the mitogen-activated protein kinase (MAPK) and phosphatidylinositol-3 kinase (PI3K) pathways. The phosphorylation of extracellular signal-regulated kinase (ERK)1/2 by Neudesin is inhibited by the pertussis toxin (PTX), an inhibitor of the Gi/Go-protein, indicating that its activity is mediated via activation of the MAP and PI3K pathways, which 
are potentially coupled with the Gi/Go-protein-coupled signaling pathway (Kimura et al., 2005) (Table 1).

Neudesin is also expressed in neural precursor cells before the appearance of neurons in mice, indicating its potential roles in neural development (Kimura et al., 2006). Neudesin significantly promotes neuronal differentiation that is mediated though activation of the protein kinase A (PKA) and PI3K pathways in cultured neural precursor cells. It also transiently promotes neural cell proliferation early in the developmental process. This proliferation is mediated through the MAPK and PKA pathways. The expression profile and activity of Neudesin indicate that it plays unique roles in neural cell proliferation and neuronal differentiation (Kimura et al., 2006). The phosphorylation of ERK, serine-threonine protein kinase AKT, and cAMP response element binding protein (CREB) is also promoted by Neudesin in neural precursor cells. However, its action is not inhibited by PTX. Neudesin increases cAMP levels in neural precursor cells, indicating that its activity is potentially exerted via the Gs protein-coupled signaling pathway and that the mechanism of action of neudesin in these cells is distinct from that in the neurons (Kimura et al., 2006). Neudesin is also abundantly expressed in cultured mouse neuroblastoma Neuro2a cells. Cell survival and proliferation are significantly decreased in Neuro2a cells by the siRNA-mediated knockdown of Neudesin (Kimura et al., 2008). Neudesin activates the MAPK and PI3K signaling pathways in cultured adipocytes and cancer cells (Kimura et al., 2009; Han et al., 2012) (Table 1).

Hemin significantly promotes the activity of Neudesin in primary cultured cells, indicating that the heme-binding domain is essential for its activity (Cahill, 2007). As Neudesin is a secreted protein, it is expected to exert its activity by binding to and activating its specific cell surface receptor. Since the activity of Neudesin is exerted via the $G$ protein-coupled signaling pathway, its receptor is expected to be a $G$ protein-coupled receptor (GPCR). Although currently unknown, the identification of Neudesin receptors will provide an important insight into the action mechanism of Neudesin.

\section{Roles of Neudesin in Neuronal Functions}

Neudesin exhibits neurotropic activity in cultured neuronal cells, therefore, it is assumed to play in vivo roles in neuronal functions. Some secreted proteins in the brain including brain-derived neurotrophic factor (BDNF), ciliary neurotrophic factor, and neuropeptide $\mathrm{Y}$ can regulate energy uptake and expenditure (Fargali et al., 2012). Neudesin was also identified through largescale screening aimed at identifying novel secreted hypothalamic proteins that regulate food intake (Byerly et al., 2013). Neudesin is strongly expressed in hypothalamic nuclei that regulate food intake. BDNF in the hypothalamus is an important regulator of appetite. The expression of Neudesin in the hypothalamus is decreased by the administration of BDNF into the lateral cerebral ventricle. The administration of Neudesin into the cerebral ventricle resulted in decreases in food intake and body weight and increases in the expression of pro-opiomelanocortin and melanocortin 4 receptor in the hypothalamus. However, the effects of Neudesin on food intake is abrogated in obese mice fed a high-fat diet (HFD), indicating the diet-dependent modulation of Neudesin functions. These findings indicate that hypothalamic Neudesin is a potential central modulator of food intake via

TABLE 1 | Activity and roles of Neudesin in neural functions, energy metabolism, and tumorigenesis.

\begin{tabular}{|c|c|c|c|}
\hline Neudesin gain-of-function & Loss-of-function & Action site & References \\
\hline \multicolumn{4}{|l|}{ NEURAL FUNCTIONS } \\
\hline Neurotrophic activity & & Cultured neuronal cells & Kimura et al., 2005 \\
\hline Differentiation activity & & Cultured neural precursor cells & \\
\hline \multirow[t]{2}{*}{ Cell proliferation activity } & & Cultured neural precursor cells & Kimura et al., 2006 \\
\hline & Inhibition of cell proliferation/survival & Cultured Neuro2a cells & Kimura et al., 2008 \\
\hline \multicolumn{4}{|l|}{ ENERGY METABOLISM } \\
\hline \multirow[t]{4}{*}{ Inhibition of adipogenesis } & & Cultured 3T3-L1 cells & Kimura et al., 2009 \\
\hline & Promotion of adipogenesis & Cultured 3T3-L1 cells & Kimura et al., 2009 \\
\hline & Resistance to HFD-induced obesity/metabolic dysfunction & Mice & Ohta et al., 2015 \\
\hline & Increased sympathetic activity & Mice & Ohta et al., 2015 \\
\hline \multicolumn{4}{|l|}{ TUMORIGENESIS } \\
\hline Invasiveness activity & & Cultured MCF cells & Han et al., 2012 \\
\hline \multirow[t]{3}{*}{ Tumorigenicity } & & Cultured MCF cells & Han et al., 2012 \\
\hline & Inhibition of cell growth & Cultured cancer cells & Stefanska et al., 2014 \\
\hline & Inhibition of invasiveness & Cultured cancer cells & Stefanska et al., 2014 \\
\hline
\end{tabular}


a regulatory circuit that may involve BDNF and melanocortin signaling (Byerly et al., 2013) (Table 1).

Neudesin knockout (KO) mice appear to be normal and fertile (Novais et al., 2013; Ohta et al., 2015). An extensive behavioral characterization (motor, emotional, and cognitive dimensions) of Neudesin KO mice revealed anxiety-like behavior. In association with the anxious phenotype, dopaminergic input was reduced and dendritic arborization was impoverished in dentate gyrus granule neurons in the ventral hippocampus. Shorter dendrites were also observed in the bed nucleus of the stria terminalis. These findings suggest the roles of Neudesin in maintaining the hippocampal anxiety circuitry (Novais et al., 2013) (Table 1).

\section{Roles of Neudesin in Energy Metabolism}

White adipose tissue (WAT) is crucially involved in energy metabolism. Obesity, which is characterized by the excessive development of WAT, is a risk factor for several metabolic diseases, including type II diabetes, hypertension, and atherosclerosis. The development of WAT involves adipogenesis and an increase in the number of cells (Hausman et al., 2001). Neudesin is also abundantly expressed in the WAT of mice. It has been shown to significantly suppress adipogenesis in cultured 3T3-L1 preadipocytes. The knockdown of Neudesin by RNA interference markedly promoted adipogenesis by suppressing activation of the MAPK pathway activation in 3T3-L1 cells. These findings suggest that Neudesin may be a negative regulator in the early stage of adipogenesis (Kimura et al., 2009) (Table 1).

Neudesin KO mice are strongly resistant to HFD-induced obesity and metabolic dysfunction. However, food intake is essentially unaffected in Neudesin KO mice fed HFD (Ohta et al., 2015). Thus, resistance to HFD-induced obesity is independent of food intake. As discussed above, the administration of Neudesin decreased food intake (Byerly et al., 2013). This discrepancy may be explained by differences in physiological analyses using Neudesin KO mice and pharmacological analyses by the administration of recombinant Neudesin.

Adipose tissue consists of two distinct types: white and brown. WAT stores excess energy as triglycerides. In contrast, brown adipose tissue (BAT) dissipates energy as heat, thereby counteracting obesity (Frontini and Cinti, 2010; Cristancho and Lazar, 2011). The sympathetic nervous system (SNS) plays crucial roles in maintaining energy homeostasis. The activated SNS stimulates lipolysis in WAT and enhances heat production in BAT by activating adrenergic signaling. Thus, the SNS is essential for regulating adipose function and the development of obesity (Tentolouris et al., 2006). Sympathetic activity was found to be significantly increased in Neudesin KO mice fed HFD, resulting in increased energy expenditure and heat production as well as fatty acid oxidation in BAT and enhanced lipolysis in WAT (Ohta et al., 2015) (Table 1). These findings indicate that Neudesin is a negative regulator of energy expenditure and could be an attractive target for the development of anti-obesity drugs.

\section{Roles of Neudesin in Tumorigenesis}

Neudesin has also been identified as GIG47 using a differential display technique to discover genes critical for breast tumorigenesis (Han et al., 2012). Neudesin is over-expressed in multiple human cancers including carcinomas of the breast, uterine cervix, malignant lymphoma, colon, lung, and skin as well as in leukemia and breast cancer cell line MCF-7. The ectopic expression of Neudesin in MCF7 cells has been shown to promote invasiveness in vitro and increase tumorigenicity in vivo. The mechanism underlying tumorigenesis may be mediated by activation of the MAPK and PI3K pathways. These findings indicate that Neudesin is involved in tumorigenesis and may be a novel target for the treatment of cancers (Table 1).

Common hypomethylated genes in many cancers are candidates for novel broad-spectrum anti-cancer and antimetastatic agents. Whole-genome mapping has identified many activated gene promoters by DNA hypomethylation in hepatocellular carcinoma (HCC) clinical samples. Neudesin is also hypomethylated and induced in HCC (Stefanska et al., 2014). The ineffectiveness of Neudesin mediated by RNA interference in different types of cancers effectively and specifically inhibited their cell growth and invasive capacities. This ineffectiveness was also found to reduce their growth as explants in mice and interfere with the AKT, WNT, and MAPK signaling pathways. These findings indicate that Neudesin is induced by hypomethylation in many cancers and is a candidate target for anti-cancer therapeutics in multiple cancer cell types (Table 1).

\section{Conclusions}

Neudesin, which is a member of the MAPR family, is a unique secreted protein with a conserved cytochrome 5-like heme/steroid-binding domain and plays multi-functional roles in neural functions, energy metabolism, and tumorigenesis. In the brain, Neudesin may be a neurotrophic factor in food intake in the hypothalamus and in maintaining the hippocampal anxiety circuitry. Neudesin $\mathrm{KO}$ mice are strongly resistant to HFD-induced obesity, indicating that Neudesin is a negative regulator of energy expenditure in peripheral tissues. Neudesin is also expressed in multiple human cancers and stimulates tumorigenesis. Further studies on Neudesin will provide useful clues for the development of treatments for metabolic diseases and cancers.

\section{Acknowledgments}

This work was supported by a Grant-in-Aid for Scientific Research from the Ministry of Education, Science, Culture and Sports of Japan (25860045 and 25460065) and Smoking Research Foundation, Japan. 


\section{References}

Bruce, A., and Rybak, A. P. (2014). CYB5D2 requires heme-binding to regulate HeLa cell growth and confer survival from chemotherapeutic agents. PLOS ONE 9:e86435. doi: 10.1371/journal.pone.0086435

Byerly, M. S., Swanson, R. D., Semsarzadeh, N. N., McCulloh, P. S., Kwon, K., Aja, S., et al. (2013). Identification of hypothalamic neuron-derived neurotrophic factor as a novel factor modulating appetite. Am. J. Physiol. Regul. Integr. Comp. Physiol. 304, R1085-R1095. doi: 10.1152/ajpregu.003 68.2012

Cahill, M. A. (2007). Progesterone receptor membrane component 1: an integrative review. J. Steroid Biochem. Mol. Biol. 105, 16-36. doi: 10.1016/j.jsbmb.2007.02.002

Chen, C., Sargent, C., Quilter, C., Yang, Z., Ren, J., Affara, N., et al. (2010). Cloning, mapping and molecular characterization of porcine progesterone receptor membrane component 2 (PGRMC2) gene. Genet. Mol. Biol. 33, 471-474. doi: 10.1590/S1415-47572010005000057

Cristancho, A. G., and Lazar, M. A. (2011). Forming functional fat: a growing understanding of adipocyte differentiation. Nat. Rev. Mol. Cell Biol. 12, 722-734. doi: $10.1038 / \mathrm{nrm} 3198$

Fargali, S., Sadahiro, M., Jiang, C., Frick, A. L., Indall, T., Cogliani, V., et al. (2012). Role of neurotrophins in the development and function of neural circuits that regulate energy homeostasis. J. Mol. Neurosci. 48, 654-659. doi: 10.1007/s12031-012-9790-9

Frontini, A., and Cinti, S. (2010). Distribution and development of brown adipocytes in the murine and human adipose organ. Cell Metab. 11, 253-256. doi: 10.1016/j.cmet.2010.03.004

Gerdes, D., Wehling, M., Leube, B., and Falkenstein, E. (1998). Cloning and tissue expression of two putative steroid membrane receptors. Biol. Chem. 379, 907-911.

Han, K. H., Lee, S. H., Ha, S. A., Kim, H. K., Lee, C., Kim, D. H., et al. (2012). The functional and structural characterization of a novel oncogene GIG47 involved in the breast tumorigenesis. BMC Cancer 12:274. doi: 10.1186/1471-2407$12-274$

Hausman, D. B., DiGirolamo, M., Bartness, T. J., Hausman, G. J., and Martin, R. J. (2001). The biology of white adipocyte proliferation. Obes. Rev. 2, 239-254. doi: 10.1046/j.1467-789X.2001.00042.x

Intlekofer, K. A., and Petersen, S. L. (2011). Distribution of mRNAs encoding classical progestin receptor, progesterone membrane components 1 and 2, serpine mRNA binding protein 1 , and progestin and ADIPOQ receptor family members 7 and 8 in rat forebrain. Neuroscience 172, 55-65. doi: 10.1016/j.neuroscience.2010.10.051

Kimura, I., Konishi, M., Asaki, T., Furukawa, N., Ukai, K., Mori, M., et al. (2009). Neudesin, an extracellular heme-binding protein, suppresses adipogenesis in 3T3-L1 cells via the MAPK cascade. Biochem. Biophys. Res. Commun. 381, 75-80. doi: 10.1016/j.bbrc.2009.02.011

Kimura, I., Konishi, M., Miyake, A., Fujimoto, M., and Itoh, N. (2006). Neudesin, a secreted factor, promotes neural cell proliferation and neuronal differentiation in mouse neural precursor cells. J. Neurosci. Res. 83, 1415-1424. doi: 10.1002/jnr.20849

Kimura, I., Nakayama, Y., Konishi, M., Kobayashi, T., Mori, M., Ito, M., et al. (2010). Neuferricin, a novel extracellular heme-binding protein, promotes neurogenesis. J. Neurochem. 112, 1156-1167. doi: 10.1111/j.14714159.2009.06522.x

Kimura, I., Nakayama, Y., Yamauchi, H., Konishi, M., Miyake, A., Mori, M., et al. (2008). Neurotrophic activity of neudesin, a novel extracellular hemebinding protein, is dependent on the binding of heme to its cytochrome b5-like heme/steroid-binding domain. J. Biol. Chem. 283, 4323-4331. doi: 10.1074/jbc.M706679200

Kimura, I., Nakayama, Y., Zhao, Y., Konishi, M., and Itoh, N. (2013). Neurotrophic effects of neudesin in the central nervous system. Front. Neurosci. 7:111. doi: 10.3389/fnins.2013.00111

Kimura, I., Yoshioka, M., Konishi, M., Miyake, A., and Itoh, N. (2005). Neudesin, a novel secreted protein with a unique primary structure and neurotrophic activity. J. Neurosci. Res. 79, 287-294. doi: 10.1002/jnr.20356

Meyer, C., Schmid, R., Scriba, P. C., and Wehling, M. (1996). Purification and partial sequencing of high-affinity progesterone-binding site(s) from porcine liver membranes. Eur. J. Biochem. 239, 726-731. doi: 10.1111/j.14321033.1996.0726u.x

Novais, A., Ferreira, A. C., Marques, F., Pêgo, J. M., Cerqueira, J. J., David-Pereira, A., et al. (2013). Neudesin is involved in anxiety behavior: structural and neurochemical correlates. Front. Behav. Neurosci. 7:119. doi: 10.3389/fnbeh.2013.00119

Ohta, H., and Itoh, N. (2012). The membrane-associated progesterone receptor (MAPR) protein family. Curr. Top. Biochem. Res. 14, 11-15.

Ohta, H., Konishi, M., Kobayashi, Y., Kashio, Y., Morichiyama, T., Matsumura, S., et al. (2015). Detection of the neurotrophic factor neudesin prevents dietinduced obesity by increased sympathetic activity. Sci. Rep. 5:10049. doi: 10.1038/srep10049

Rohe, H. J., Ahmed, I. S., Twist, K. E., and Craven, R. J. (2009). PGRMC1 (progesterone receptor membrane component 1): a targetable protein with multiple functions in steroid signaling, P450 activation and drug binding. Pharmacol. Ther. 121, 14-19. doi: 10.1016/j.pharmthera.2008.09.006

Stefanska, B., Cheishvili, D., Suderman, M., Arakelian, A., Huang, J., Hallett, M., et al. (2014). Genome-wide study of hypomethylated and induced genes in patients with liver cancer unravels novel anticancer targets. Clin. Cancer Res. 20, 3118-3132. doi: 10.1158/1078-0432.CCR-13-0283

Tentolouris, N., Liatis, S., and Katsilambros, N. (2006). Sympathetic system activity in obesity and metabolic syndrome. Ann. N.Y. Acad. Sci. 1083, 129-152. doi: 10.1196/annals.1367.010

Xie, Y., Bruce, A., He, L., Wei, F., Tao, L., and Tang, D. (2011). CYB5D2 enhances HeLa cells survival of etoposide-induced cytotoxicity. Biochem. Cell Biol. 89, 341-350. doi: 10.1139/o11-004

Conflict of Interest Statement: The authors declare that the research was conducted in the absence of any commercial or financial relationships that could be construed as a potential conflict of interest.

Copyright (C) 2015 Ohta, Kimura, Konishi and Itoh. This is an open-access article distributed under the terms of the Creative Commons Attribution License (CC BY). The use, distribution or reproduction in other forums is permitted, provided the original author(s) or licensor are credited and that the original publication in this journal is cited, in accordance with accepted academic practice. No use, distribution or reproduction is permitted which does not comply with these terms. 